\title{
COVID-19 en Pediatría: Manifestaciones Clínicas
}

COVID-19 in Pediatrics: Clinical Manifestations

*Marlon Alexander Cerna Márquez, **Larissa Maydelin

Contreras Martínez, **María Verónica Lazo Quevedo.

\section{RESUMEN}

Los coronavirus son importantes patógenos humanos y animales. A finales de 2019, se identificó un nuevo coronavirus como la causa de un grupo de casos de neumonía en Wuhan, una ciudad de la provincia de China de Hubei. Se extendió rápidamente, dando como resultado una epidemia en toda China, seguida de un número de casos crecientes en otros países del mundo. El virus que causa COVID-19 se denomina SARS-CoV-2 (Coronavirus 2 del Síndrome Respiratorio Agudo Severo). Los casos pediátricos actualmente, son menos frecuentes que los adultos, con formas de presentación diferente y síntomas más leves y con mejor pronóstico. La mayoría tuvo exposición a miembros del hogar con COVID-19 confirmado. Los niños tienen la misma probabilidad que los adultos de infectarse. En Honduras hasta el 17 Junio de 2020 los casos reportados en el grupo de 0-18 años representan el 6.2\%. Los pacientes pediátricos puede presentar cuadros que varían desde un cuadro leve hasta falla multiorgánica. La infección por esta nueva cepa de coronavirus genera una amplia gama de manifestaciones clínicas que van desde afectación respiratoria, dermatológica, neurológica, oftálmicas, gastrointestinal, hepática, renal, cardiaca y hematológicas. Afortunadamente la mayoría de los paciente pediátricos son asintomáticos o con síntomas respiratorios altos, comprendiendo

*Residente de primer año de Cirugía Pediátrica en el Instituto Nacional de Pediatría, UNAM (Universidad Nacional Autónoma de México).

**Residente de segundo año de Neumología Pediátrica en el Instituto Nacional de Pediatría, UNAM (Universidad Nacional Autónoma de México)

Dirigir correspondecia: larissa_macon@hotmail.com Recibido: 5 de Mayo de 2020 Aceptado: 10 de Septiembre de 2020 aproximadamente el $90 \%$ de los casos, los cuales se recuperan en 1-2 semanas después del inicio de los síntomas. En los casos neonatales la mayoría (>90\%) de recién nacidos de madres con COVID-19 son asintomáticos y no presentan complicaciones al nacer, incluidos aquellos en donde se corrobora infección por SARS-CoV-2.

PALABRAS CLAVE: COVID-19, Pandemia, Pediatría, signos y síntomas, Infecciones por Coronavirus.

\section{ABSTRACTS}

Coronaviruses are important human and animal pathogens. In late 2019, a new coronavirus was identified as the cause of a group of pneumonia cases in Wuhan, a city in China's Hubei province. It spread rapidly, resulting in an epidemic across China, followed by a growing number of cases in other countries of the world. The virus that causes COVID-19 is called SARS-CoV-2 (Coronavirus 2 of Severe Acute Respiratory Syndrome). Currently, pediatric cases are less frequent than adults, with different presentation forms and milder symptoms and with a better prognosis. Most had exposure to household members with confirmed COVID-19. Children are just as likely as adults to get infected. In Honduras until June 17,2020 , the cases reported in the 0 - 18 age group represent $6.2 \%$. Pediatric patients may present with symptoms that vary from mild symptoms to multi-organ failure. Infection with this new strain of coronavirus generates a wide range of clinical manifestations, ranging from respiratory, dermatological, neurological, ophthalmic, gastrointestinal, hepatic, renal, cardiac and hematological involvement. Fortunately, most pediatric patients are asymptomatic or with upper 
respiratory symptoms, comprising approximately 90\% of cases, which recover within 1-2 weeks after the onset of symptoms. In neonatal cases the majority (>90\%) of newborns of mothers with COVID-19 are asymptomatic and do not present complications at birth, including those where SARS-CoV-2 infection is corroborated.

KEY WORDS: COVID-19, Pandemics, Pediatrics, Signs and Symptoms, Coronavirus Infections,

\section{INTRODUCCIÓN}

El Coronavirus se compone de una gran familia de virus que son comunes en los seres humanos y en los animales (murciélagos, camellos, vacas y gatos). ${ }^{(1)}$ A finales del 2019, se identificó un nuevo Coronavirus como la causa de un grupo de casos de neumonía en Wuhan, una ciudad de la provincia de Hubei en China. Se extendió rápidamente, dando como resultado un epidemia en toda China, seguida de un número creciente de casos en otros países del mundo. ${ }^{(2)}$ En febrero 2020, la Organización Mundial de la Salud (OMS), designó la enfermedad como COVID-19, que significa enfermedad por Coronavirus 2019(3) El virus que causa COVID-19 se denomina SARS-CoV-2 (Coronavirus 2 del Síndrome Respiratorio Agudo Severo). ${ }^{(4)}$ Los casos pediátricos actualmente, son menos frecuentes que los adultos. Los datos aportados por Centro de Enfermedades de los Estados Unidos (CDC) a inicios de abril 2020 describen que un $1.7 \%$ de los casos reportados corresponden a menores de 18 años. ${ }^{(5)}$ De la misma forma, en los reportes de China se observa que un $2 \%$ de los casos corresponden a menores de 19 años y en un reporte italiano un $1.2 \%{ }^{(6)}$ En el reporte del MINSAL del 27 de Abril los pacientes entre 0-4 años corresponde a un 1.3\% de los casos, los pacientes de 10-14 años al 1.8\% de los casos y los pacientes de 15-19 años el 3\% de los notificados. (7) En Honduras hasta el 17 Junio de 2020 los casos reportados en el grupo etario de 0-18 años representan el $6.2 \% .{ }^{(8)}$

Los pacientes pediátricos puede presentar cuadros que varían desde un cuadro leve y de vías respiratorias altas hasta un cuadro de neumonía grave con sepsis. ${ }^{(9,10)}$ La infección por esta nueva cepa de coronavirus genera una amplia gama de manifestaciones clínicas. Los primeros datos sobre el comportamiento de la enfermedad ocasionada por este agente viral se obtuvieron a través de un informe del Centro Chino para el Control y Prevención de Enfermedades donde se analizó un total de 72,314 casos registrados, de lo cuales 44,672 se catalogaron como positivos y permitieron generar una clasificación a esta entidad clínica usando signos y síntomas, índice de oxigenación y estudios de imagen. ${ }^{(11)}$

\section{BÚSQUEDA DE DATOS}

La búsqueda bibliográfica de este artículo se basó principalmente en Internet, utilizando PubMed, UptoDate, Lancet, Elsevier, JAMA, NEJM y Google Académico. Se compiló una lista de términos y frases de búsqueda para centrarse en los temas generales de ' manifestaciones clínicas', 'Pediatría', 'COVID-19', ' 2019-nCoV ' o ' SARSCoV-2 ', sin imponer restricciones de fecha o año, ubicaciones, diseño del estudio, objetivo del estudio o criterios de inclusión/exclusión. Revisamos todos los artículos publicados hasta el 17 de Junio de 2020.

\section{Período de incubación}

Similar a lo observado en adultos se describen períodos de incubación de van desde 2 a 10-14 días posteriores a la exposición (promedio 6.5 días, mediana 3-7 días) y la mayoría de los casos ocurren de 4-5 días después de la exposición. $(9-12,21)$ En un estudio de 1099 pacientes con COVID-19 sintomático confirmado, la mediana del período de incubación fue de cuatro días (rango intercuartil de dos a siete días)

\section{Espectro de Severidad del COVID-19}

Desde el punto de vista clínico es posible clasificar los pacientes pediátricos con COVID-19 en las siguientes categorías: (Ver Tabla 1) 


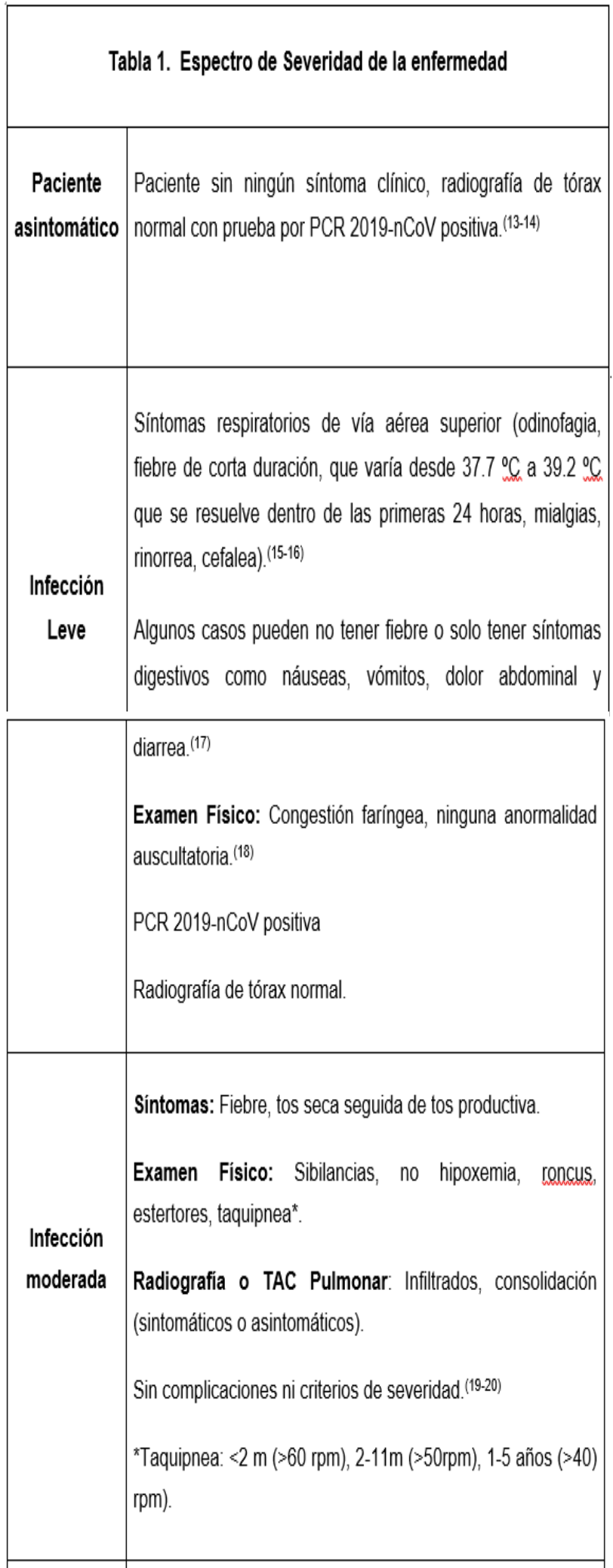

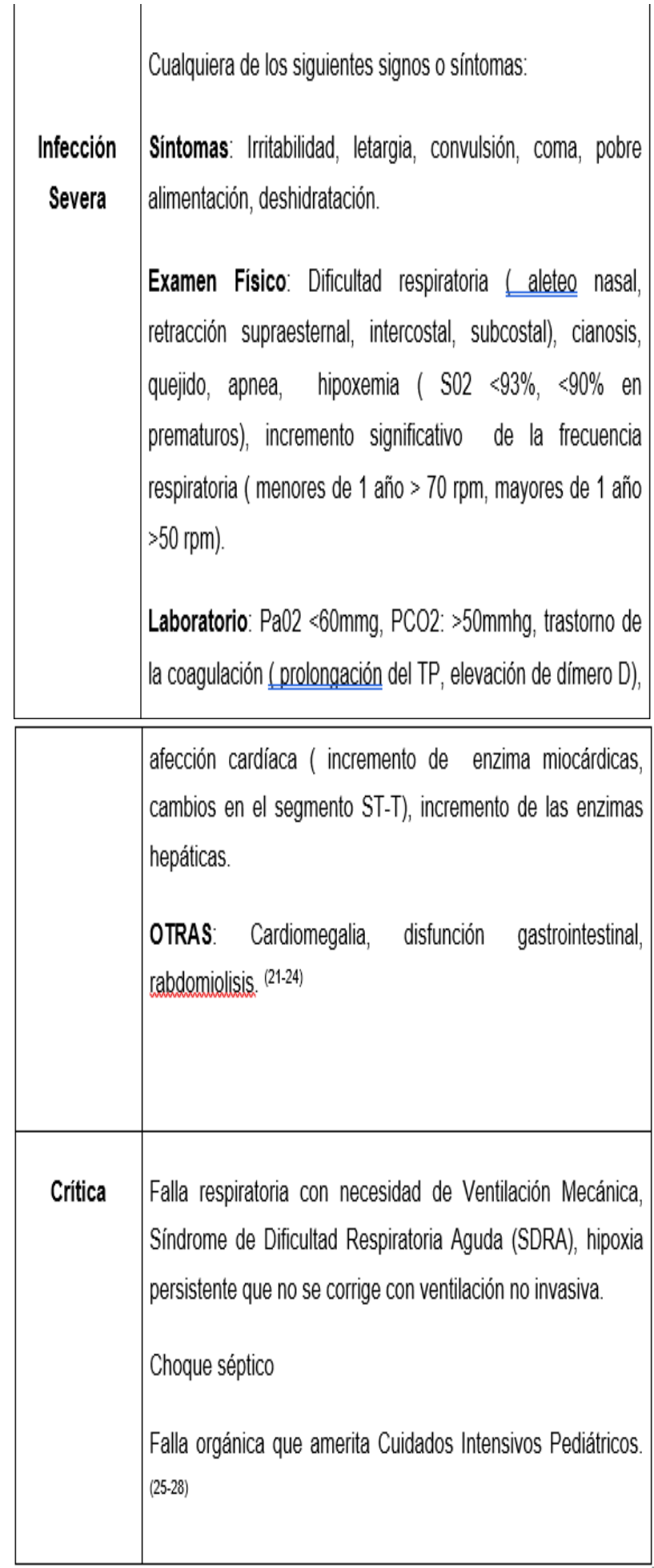


Afortunadamente la mayoría de los paciente pediátricos son asintomáticos o con síntomas respiratorios altos, comprendiendo aproximadamente el 90\% de los casos, los cuales se recuperan en 1-2 semanas después del inicio de los síntomas. ${ }^{(27-29)}$ $\mathrm{Al}$ organizarlos por grupos de edad, los menores de 1 año presentan enfermedad severa (10\%), el grupo de 1-5 años (7.3\%), el grupo de 6-10 años (4.2\%), el grupo de 11-15 años (4.1\%) y en el grupo de 16-17 años (3\%). ${ }^{(30)}$

Las condiciones subyacentes más comúnmente reportadas en la población pediátrica con $\mathrm{CO}$ VID-19 en Estados Unidos fueron la enfermedad pulmonar crónica (incluyendo asma moderada a severa) y la enfermedad cardiovascular e inmunosupresión. ${ }^{(32,33)}$ Otras afecciones médicas que pueden aumentar el riesgo de enfermedad grave incluyen: ${ }^{(34-37)}$

$\checkmark$ Trastornos de la sangre.

$\checkmark$ Enfermedad renal crónica en terapia sustitutiva renal.

$\checkmark$ Enfermedad hepática crónica.

$\checkmark$ Embarazo.

$\checkmark$ Trastornos endocrinos.

$\checkmark$ Cáncer.

$\checkmark$ Obesidad severa (IMC $\geq 40 \mathrm{~kg} / \mathrm{m} 2)$.

\section{¿Cómo se presenta clinicamente el CO- VID-19 en niños?.}

Los casos positivos por COVID-19 en pacientes pediátricos, hasta la fecha se ha presentado en menor porcentaje que en los adultos y con síntomas más leves y con mejor pronóstico, según el informe de situación COVID-19 emitido por la Organización Mundial de la Salud (OMS). ${ }^{(38)}$ Un estudio publicado a principios de marzo de 2020 sugiere que los niños tienen la misma probabilidad que los adultos de infectarse con el COVID-19, pero es menos probable que presenten síntomas o desarrollen síntomas graves. ${ }^{(39)}$

Según la CDC para el mes de mayo de 2020 en los Estados Unidos, el 2\% de los casos confirmados de COVID-19 fueron menores de 18 años, en China el $2.2 \%$ y en Italia el 1,2\% de los casos correspondió a este grupo etario. En Honduras según el Despacho de comunicaciones y estrategia presidencial, no se han reportado casos positivos en menores de 20 años. $(8,40-43)$

De los casos reportados, la mayoría tuvo exposición a miembros del hogar con COVID-19 confirmado. ${ }^{(4)}$

Aunque la mayoría de los pacientes con COVID-19 manifiestan fiebre y síntomas del tracto respiratorio, la infección también puede afectar a otros órganos/sistemas y presentarse con manifestaciones extra respiratorias, que incluyen enfermedades cardíacas, gastrointestinales, hepáticas, renales, neurológicas, olfativas, gustativas, oculares, cutáneas y hematológicas. Los pulmones son los órganos más afectados porque el virus accede a las células huésped a través de la enzima convertidora de angiotensina 2 (ACE2), que es más abundante en las células alveolares tipo II. El virus utiliza una glucoproteína de superficie, llamada "spike", para unirse a ACE2 y entrar en la célula huésped. ${ }^{(40)}$ Por lo tanto, las manifestaciones respiratorias siguen siendo los síntomas más comunes. Los signos y síntomas evidenciados pueden ser similares a los presentados en otras infecciones respiratorias virales como resfrío común, laringuitis, bronquitis, neumonía. ${ }^{(45)}$

Dentro de las principales manifestaciones clínicas en niños se encuentra fiebre $(91 \%-100 \%)$, tos seca $(43 \%-80 \%)$, mialgia $(10 \%-40 \%)$, cefalea (14\% -40\%), no necesariamente presentes en todos los casos y al inicio de la enfermedad. También pueden presentar síntomas de la vía aérea superior como congestión nasal, secreción nasal $(33 \%-60 \%)$ y dolor de garganta. ${ }^{(46-48)}$

Un estudio informó que entre 134 niños diagnosticados, 89 presentaron fiebre (11 niños fiebre alta, 17 fiebre baja y media). La duración de la fiebre fue principalmente de 1 a 2 días, y la más larga fue de 8 días. ${ }^{(49)}$

En el Hospital de niños en Wuhan China, de un total de 171 niños que tuvieron prueba positiva para COVID-19, monitorizados hasta el 8 de marzo de 2020, el 15.8\% fueron asintomáticos, el $19.3 \%$ presentaron infección del tracto respiratorio superior y el $64.9 \%$ tuvieron neumonía. Dentro de los síntomas y signos más frecuen- 
tes estuvieron la tos $(83 \%)$, eritema faríngeo (79\%), taquicardia (72\%), fiebre (71\%), taquipnea $(49 \%)$, diarrea (15\%), fatiga (13\%); solo el $4 \%$ de pacientes tuvo hipoxemia en la valoración inicial. ${ }^{(50)}$

De una pequeña serie de casos de 9 madres que fueron infectadas con COVID-19, hasta la fecha, no hay evidencia de que pueda transmitirse verticalmente al bebé. ${ }^{(51)}$

En los informes de casos neonatales la mayoría (>90\%) de recién nacidos de madres con COVID-19 son asintomáticos y no presentan complicaciones al nacer, incluidos aquellos en donde se corrobora infección por SARS-CoV-2. Muestran principalmente síntomas de infecciones del tracto respiratorio, como tos y secreción nasal y gastrointestinales, así como nacimiento pretérmino, distrés respiratorio y sepsis. ${ }^{(52-55)}$

Tiago H. y colaboradores realizaron una revisión sistemática de 38 estudios entre diciembre 2019 y abril de 2020 (1.117 niños); de los cuales 14.2\% eran asintomáticos, $36.3 \%$ eran leves, $46.0 \%$ eran moderados, $2.1 \%$ eran severos y $1.2 \%$ eran críticos. El síntoma más prevalente fue fiebre (47.5\%), seguido de tos (41.5\%), síntomas nasales (11.2\%), diarrea $(8.1 \%)$ y náuseas/vómitos (7.1\%). Ciento cuarenta y cinco (36.9\%) niños fueron diagnosticados con neumonía y 43 (10.9\%) infecciones de las vías aéreas superiores. Los casos severos muestran disnea (10\%-14\%), que puede progresar rápidamente a síndrome de dificultad respiratoria aguda (SDRA), shock séptico, acidosis metabólica, disfunción de la coagulación y falla multiorgánica. ${ }^{(47-48)}$

Los casos de síndrome respiratorio agudo severo (SARS-CoV-2), aumentaron gradualmente. En la etapa inicial, se produjo principalmente en adultos. Hay pocos casos reportados en niños. La mayoría de los niños tienen síntomas clínicos leves, con recuperación dentro de 1-2 semanas. Sin embargo, las anomalías en la tomografía computarizada de tórax pueden persistir durante varios meses. Algunas manifestaciones extra respiratorias, como la afectación ocular y gastrointestinal, pueden ser causadas por la invasión directa del COVID-19; otras como la afectación muscular cardíaca, lesión renal aguda, daño hepático, trastornos de la coagulación y complicaciones trombóticas, podrían estar asociadas a un mal pronóstico. Se ha asociado que cifras aumentadas de LDH son más propensos a causar lesiones cardiopulmonares e inflamación en niños, aunque la inflamación pulmonar en niños es menos severa que en adultos. ${ }^{(56)}$ Las características particulares de laboratorio que también se asocian con peores resultados incluyen: linfopenia, enzimas hepáticas elevadas, marcadores inflamatorios elevados (PCR y ferritina), Dímero $\mathrm{D}$ elevado, tiempo elevado de protombina (TP), troponina elevada, CPK elevada. ${ }^{(54)}$

Las alteraciones a nivel del gusto y del olfato (disgeusia, ageusia, hiposmia) se explican están asociados con infecciones virales, y el COVID-19 no es una excepción. Un estudio in vitro mostró que el receptor ACE2 se expresa ampliamente en las células epiteliales de la mucosa oral, lo que sugiere un posible mecanismo patogénico para la asociación entre COVID-19 y los trastornos olfativos y gustativos. Un estudio de vigilancia que incluyó pacientes con COVID-19 leve a moderado en 12 hospitales europeos informó que $85.6 \%$ (357/417) y $88.8 \%(342 / 385)$ de los pacientes tenían disfunciones olfativas y gustativas, respectivamente. ${ }^{(57-59)}$

Los síntomas gastrointestinales son comunes en pacientes con COVID-19 y presentes con mayor frecuencia en la población pediátrica $(57 \%)$ y pueden estar presentes en ausencia de síntomas respiratorios. ${ }^{(40,41,50,53)}$ Zhang et al, informaron que ACE2 se expresó altamente en las células epiteliales esofágicas y los enterocitos absorbentes del íleon y el colon, lo que sugiere una posible transmisión fecal. Yuan Tian y colaboradores revisaron las características gastrointestinales y los resultados de las pruebas fecales en COVID-19 a partir de informes de casos y estudios clínicos retrospectivos reportados desde el brote (2023 casos); concluyeron que los síntomas gastrointestinales más frecuentes fueron anorexia 39.9\%$50.2 \%$, diarrea $2 \%-49.5 \%$, vómitos $3.6 \%-66.7 \%$, náuseas 1\%-29.4\%, dolor abdominal 2.2\%-6\% y hemorragia gastrointestinal 4\%-13.7\%. La diarrea fue el síntoma gastrointestinal más común 
en niños y adultos, con una duración media de $4.1 \pm 2.5$ días, y se observó antes y después del diagnóstico. El vómito fue más prominente en los niños. ${ }^{(44,46)}$

Las manifestaciones cutáneas están presentes en varias infecciones virales. En una serie de 88 pacientes infectados con COVID-19 en Italia, Recalcati mostró que el 20,5\% de pacientes desarrollaron lesiones cutáneas caracterizadas por erupciones eritematosas (la manifestación más común), urticaria, vesículas. Además, la acroisquemia se presentó con cianosis de dedo, ampollas cutáneas y gangrena seca en pacientes críticos. ${ }^{(43)}$

En una serie de casos que incluyó a 38 pacientes con COVID-19, 12 pacientes tuvieron manifestaciones oculares, como congestión conjuntival, epífora o quemosis, y estos signos aparecieron con frecuencia en casos con manifestaciones sistémicas más grave, al momento no se dispone de datos estadísticos sobre su presencia en la población infantil. ${ }^{(60)}$

El 6 de mayo de 2020, autores de Londres, Inglaterra informaron características clínicas y de laboratorio de un grupo de 8 niños con shock hiperinflamatorio, positivos para COVID-19. ${ }^{(44)}$ Las características clínicas de estos casos comparten características con síndrome de shock tóxico, enfermedad de Kawasaki, al que se denominó síndrome inflamatorio multisistémico (SIM). Desde el mes de abril ha habido un número creciente de informes mundiales de niños con SIM con fiebre persistente, diarrea y erupción cutánea variable, conjuntivitis y edema de extremidades, a menudo asociado con una enfermedad grave que incluye shock y disfunción miocárdica; síntomas y signos clásicos de la enfermedad de Kawasaki, sin embargo, estudios demuestran ciertas diferencias entre esta patología y el síndrome inflamatorio multisistémico como la disfunción cardíaca con troponina y BNP elevados, enteropatía presentada con más frecuencia que en el síndrome de Kawasaki y relativa trombocitopenia en lugar de trombocitosis. ${ }^{(61-63)}$

La afección del sistema nervioso se puede explicar por el neurotropismo e ingreso del COVID-19 al sistema nervioso central (SNC) por diseminación vascular sistémica o través de la placa cribiforme del hueso etmoides. Se han descrito manifestaciones leves como dolor de cabe$\mathrm{za}$, mareo, anosmia hasta convulsiones, derrames cerebrales y síndrome de Guillain-Barré. ${ }^{(39-42}$ ) Un estudio temprano de la ciudad de Wuhan, China, de 214 pacientes hospitalizados con COVID-19 informó que más de un tercio (36.4\%) de los pacientes tenían algún grado de afectación neurológica. El dolor de cabeza y los mareos fueron las dos manifestaciones más reportadas del SNC con $17 \%$ y $13 \%$, respectivamente. La incidencia de secuelas neurológicas más graves, como los accidentes cerebrovasculares y las convulsiones, fue baja al 3\% y al 0,5\%, respectivamente. Las personas con infecciones graves eran más propensas a sufrir complicaciones neurológicas. ${ }^{(64-65)}$

Recientemente, se han informado algunos casos en Italia de síndrome de Guillain-Barré (SGB) y Miller Fisher (MF), después de la infección por COVID-19, caracterizados por polirradiculoneuritis con debilidad de las extremidades inferiores, parestesia, diplejía facial seguida de ataxia, la tetraparesia o tetraplejia flácida generalizada evolucionó durante un período de 36 horas a 4 días. El intervalo entre el inicio de los síntomas de Covid-19 y los primeros síntomas del síndrome de Guillain-Barré varió de 5 a 10 días. Presentaron buena respuesta a la inmunoglobulina lo que indica una naturaleza inmunomediada, no se dispone de reportes de casos en pacientes pediátricos. ${ }^{(56-57)}$.Las infecciones secundarias no parecen ser complicaciones comunes de COVID-19 en general, aunque los datos son limitados. ${ }^{(45-48)}$ En una revisión de nueve estudios, principalmente de China, la tasa informada de coinfecciones bacterianas o fúngicas fue del $8 \%$; estos incluyeron principalmente infecciones respiratorias y bacteriemia. ${ }^{(50)}$ Varios informes han descrito la presunta aspergilosis invasiva entre pacientes inmunocompetentes con SDRA de COVID-19, aunque la frecuencia de esta complicación es incierta. ${ }^{(45)}$ Los estudios de autopsia han observado ARN de SARS-CoV-2 detectable ( $y$, en algunos casos, antígeno) en los riñones, el hígado, el corazón, el cerebro y la sangre además 
de las muestras del tracto respiratorio, lo que sugiere que el virus se disemina sistémicamente en algunos casos; si los efectos citopáticos virales directos en estos sitios contribuyen a las complicaciones observadas es incierto. ${ }^{(45)}$

\section{Características Clínicas del COVID-19 en Recién Nacidos, Lactantes y Niños}

En la serie de casos COVID-19 más grande hasta la fecha en China continental

(72,314 casos, actualizados hasta el 11 de febrero de 2020), 416 casos (1\%) tuvieron menos de 10 años, pero no se informó la incidencia en recién nacidos:

sin embargo, no hubo muertes ocurrió en este grupo de edad, mientras que la

tasa general de letalidad (CFR) fue del 2.3\%. ${ }^{(66)}$ La serie de casos con 34 niños proporciona la mayoría de los detalles clínicos: ninguno de los niños tenía una enfermedad subyacente, el 65\% tenía síntomas respiratorios comunes, el 26\% tenía una enfermedad leve y el 9\% eran asintomáticos. Los síntomas más comunes fueron fiebre $(50 \%)$ y tos $(38 \%) .{ }^{(67)}$ En la serie de casos de 20 niños, la presentación fue con fiebre baja a moderada o sin fiebre, rinitis, tos, fatiga, dolor de cabeza, distensión abdominal, diarrea y, en casos más graves, con disnea, cianosis y mala alimentación. ${ }^{(68)}$ En la serie de 9 lactantes, solo 4 tenían fiebre. Un lactante estaba asintomático. (69) También se han descrito niños asintomáticos adicionales infectados con SARS-CoV-2 fuera de estas series de casos (p. Ej., Un niño asintomático de 10 años con opacidades pulmonares de vidrio esmerilado radiológicas en la TC de tórax). ${ }^{(66-68)}$ La mayoría de los niños infectados se recuperan de 1 a 2 semanas después del inicio de los síntomas. Aunque los casos de COVID-19 han sido raros en lactantes y recién nacidos. Sin embargo, la mayoría de los niños infectados con SARSCoV-2 hasta ahora han sido parte de un brote de grupo familiar ( $100 \%$ en la serie de lactantes, en la que otro miembro de la familia tenía síntomas antes que los lactantes en todos los casos; $82 \%$ en la serie de casos de 34 niños; (67) Se han notificado casos de infección neonatal con COVID-19, el más joven con infección confirmada ha sido 30 horas después del nacimiento. El daño potencial sigue siendo en gran medida desconocido en los recién nacidos, especialmente prematuros, estos necesitan una observación más cercana y cautelosa, porque es más probable que presenten síntomas insidiosos y no específicos como letargo e incluso deshidratación. ${ }^{(70,71)}$ Actualmente no hay evidencia de infección intrauterina causada por transmisión vertical de madre a hijo. Los recién nacidos puede adquirir la infección a través del contacto cercano con madres infectadas. ${ }^{72-}$ 74) Generalmente los lactantes y recién nacidos presentan síntomas atípicos, que se manifiestan con vómitos, diarrea y otros síntomas gastrointestinales, o solo asma y falta de aliento; pero se han observado resultado favorables. ${ }^{(32,33)}$ El primer caso grave de infección infantil reportado en Wuhan, China, comenzó con síntomas gastrointestinales, no mostró manifestaciones respiratorias tempranas obvias, pero progresó rápidamente al síndrome de dificultad respiratoria aguda. ${ }^{(34)}$

\section{Características Clínicas del COVID-19 en Pediatría en comparación con los Adultos}

Generalmente la presentación de los síntomas en los niños comienza después de un diagnóstico confirmado en uno de los miembros de la familia, mientras que en los adultos las vías de infección son diversas. De acuerdo con publicaciones recientes, ${ }^{(75-80)}$ La fiebre y la tos fueron los síntomas dominantes tanto en niños como en adultos. Se observó que los pacientes adultos presentan frecuentemente mialgia y artralgia, rara vez desarrollaban signos y síntomas intestinales (p. Ej., diarrea y / o vómitos), mientras que aproximadamente el $57.1 \%$ de los niños pacientes tenían diarrea. ${ }^{(79,80)}$ Algunos estudiosos especulan que el virus COVID-19 puede interactuar con el receptor ACE2 en el tracto gastrointestinal, deteriorar la barrera de la membrana mucosa intestinal y aumentar la producción de citocinas inflamatorias. ${ }^{(81)}$ Por lo tanto, si una mayor tasa de síntomas gastrointestinales en el grupo de niños está relacionada con una mayor expresión de ACE2 en el tracto gastrointestinal. ${ }^{(1-3)}$ 
Del mismo modo, la duración de la fiebre en adultos fue mayor que en niños. Esto se debió, al menos en parte, a las diferencias en las respuestas inmunes, especialmente la respuesta inmune específica. Los adultos parecían más susceptibles a una respuesta inmune perjudicial. ${ }^{(4-6)}$

\section{¿Por qué en los niños los síntomas de COVID-19 es menos agresivo?}

El comportamiento menos agresivo del COVID-19 en los niños todavía no está totalmente claro, esto significa que los niños y jóvenes que no tienen afecciones subyacentes, como la función pulmonar deteriorada o inmunosupresión, tienen un riesgo mucho más bajo de formas graves de COVID-19 que otros grupos de edad. ${ }^{(82)}$ Las razones de esta enfermedad leve en niños sigue siendo difícil de dilucidar, se ha informado de varias teorías que podrían proteger a estos pacientes de una enfermedad grave, una de las teorías plantea que la proteína S de SARS-CoV-2 que media la entrada a las células epiteliales del tracto respiratorio humano debe interactuar con el receptor de la enzima convertidora de angio-

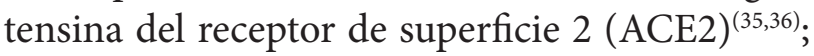
este receptor se expresa en las vías respiratorias, los pulmones y los intestinos, pero no en las células inmunes. El número de estos receptores es significativamente menor en los niños en comparación con los adultos ${ }^{(22)}$ y existe mayor concentración en el pulmón infantil, lo que pudiera explicar que la población pediátrica presente menos síntomas clínicos y disfunción orgánica que los adultos, además de lesiones pulmonares aún en pacientes pediátricos asintomáticos; sin embargo, otra posible teoría es la menor intensidad de la respuesta inmune frente a la infección viral en los niños, ocasiona menor respuesta inflamatoria frente al COVID-19, se cree que la tormenta de citoquinas es importante en la patogenia de las infecciones graves por COVID-19 ${ }^{(31)}$ y menor enfermedad clínica, este patrón parece correlacionarse inversamente con la edad. ${ }^{(28)}$ Además, la exposición recurrente al virus Sincitial respiratorio puede inducir más niveles de inmunoglobulina contra la nueva infección viral en compara- ción con los adultos. ${ }^{(29,30)}$ Otto y col. demostraron que los niños que habían recibido la vacuna combinada contra la difteria, la tos ferina, el tétanos y la poliomielitis dentro del tercer mes de vida tenían significativamente menos infecciones sintomáticas que aquellos con inmunizaciones tardías o parciales, tal vez debido a una vacunación asociada a un aumento no específico de la actividad inmunológica (por ejemplo, interleucina 2 mediado) u otros factores aún desconocidos. (83) Otra posibilidad es la presencia de otros virus simultáneas en la mucosa de los pulmones y las vías respiratorias, que son comunes en los niños pequeños, podrían limitar el crecimiento de COVID-19 mediante interacciones directas de virus a virus y la competencia por los receptores. ${ }^{(84)}$ Esto encaja con los datos emergentes de la pandemia actual, que ha indicado un vinculo entre la cantidad de copias virales y la gravedad del COVID-19. ${ }^{(85)}$ Aunque los niños tienden a tener formas leves de COVID-19, eso no significa que debamos ignorar a aquellos que tienen la enfermedad. Aún debemos tomar medidas para evitar que se infecten si queremos mitigar la pandemia. Los niños probablemente pueden transmitir virus, y se ha descubierto que albergan grandes cantidades de virus, incluso sin mostrar síntomas. También debemos tener en cuenta que los virus pueden persistir en las heces mucho después de que estén ausentes de las secreciones nasofaríngeas. ${ }^{(86)}$

\section{REFERENCIAS BIBLIOGRÁFICAS}

1. Organización Mundial de la Salud. Observaciones del Director General en los medios de comunicación en 2019-nCoV el 11 de febrero de 2020. [Revista en internet] 2020 [Citado, 29 Mayo 2020]. Disponible en: https://www.who. int/dg/speeches/detail/who-director-general-sremarks-at-the-media-brie fi ng-on-2019-ncovon-11-february-2020

2. Gorbalenya AE, Baker SC, Baric RS, et al. Coronavirus agudo severo relacionado con el síndrome respiratorio: la especie y sus virus, una declaración del Grupo de Estudio Coronavirus. 
bioRxiv 2020. [Revista en internet] 2020 [Citado, 18 Mayo 2020] Disponible en: https://www.biorxiv.org/content/10.1101/2020.02.07.937862v1.

3. Centros de Control y Prevención de Enfermedades. 2019 Nuevo coronavirus, Wuhan, China. Información para profesionales de la salud. [Revista en internet] 2020 [Citado, 14 Mayo 2020] Disponible en: https://www.cdc.gov/coronavirus/2019-nCoV/hcp/index.html.

4. Tang X, Wu C, Li X y col. Sobre el origen y la evolución continua del SARS-CoV-2. National Science Review. [Revista en internet] 2020 [Citado, 31 Mayo 2020].

5. Bialek S., Gierke R., Hughes M., McNamara L., Pilishvil T., Skoff T. COVID-19 Responde Team. Prevention, Morbidity and Mortality Weekly Report Center for Disease Control. Coronavirus Disease 2019 in Children-United States, February 12-April 12, 2020. [Revista en internet] 2020 [Citado, 30 Mayo 2020].

6. Lubvigsson JF. Systematic review of COVID-19 in children shows milder cases and a better prognosis tan adults. Acta Paeditr. [Revista en internet] 2020 [Citado, 01 Junio 2020]. Disponible en: DOI: 10.1111/apa.15270. PMID:32202343.

7. Duodécimo Informe Epidemiológico de la enfermedad por COVID-19 Departamento de Epidemiología. Minsal, 28 abril 2020. [Revista en internet] 2020 [Citado, 24 Mayo 2020] Disponible en: https:/www.minsal.cl/duodécimo-informe-epidemiologico-covid-19/.

8. Covid19honduras.org. Despacho de Comunicaciones y Estrategia Presidencial (Internet). Coronavirus COVID-19 en Honduras. (Actualizada el 17 de Junio de 2020; consultada el 17 de Junio 2020), Disponible en: https://covid19honduras. org/

9. Asociación Española de Pediatría (AEP), Sociedad Española de Infectología Pediátrica (SEIP), Sociedad Española de Urgencias de Pe- diatría (SEUP), Sociedad Española de Cuidados Intensivos Pediátricos (SECIP). Documento de manejo clínico del paciente pediátrico con infección por SARS-CoV2. [Revista en internet] 2020 [Citado, 25 Mayo 2020].

10. Cai J, Xu J, Lin D, Yang Z, Xu L, Qu Z, et al. A Case series of children with 2019 novel coronavirus infection: clinical and epidemiolical features, clinical Infectious Diseases, ciaa198. [Revista en internet] 2020 [Citado, 16 Mayo 2020] Disponible en: https:/doi.org/10.1093/cid/ ciaa 198.

11. Gallagher TM, Buchmeier MJ. Coronavirus spike proteins in viral entry and pathogenesis. Virology. [Revista en internet] 2020 [Citado, 12 Mayo 2020];279:371-4.

12. Jin Y., Cai L., Cheng Z., et al. A rapid advice guideline for the diagnosis and treatment of 2019 novel coronavirus (2019-nCoV) infected pneumonia. Miliatery Med Res. [Revista en internet] 2020 [Citado, 3 Junio 2020]. Disponible en: https://doi.org/10.1186/s40779-020-0233-6.

13. United States Centers for Disease Control and Prevention. Coronavirus disease (COVID-19). People who are at higher risk for severe illness. [Revista en internet] 2020 [Citado, 10 Mayo 2020] Disponible en: https://www.cdc.gov/ coronavirus/2019-ncov/need-extra-precautions/ people-at-higher-risk.html.

14. CDC COVID-19 Response Team. Preliminary Estimates of the Prevalence of Selected Underlying Health Conditions Among Patients with Coronavirus Disease 2019 - United States, February 12-March 28, 2020. MMWR Morb Mortal Wkly Rep. 2020;69(13):382-386. [Revista en internet] 2020 [Citado, 13 Mayo 2020]. DOI:10.15585/mmwr.mm6913e2.

15. Zhu N, Zhang D, Wang W, et al. Un nuevo coronavirus de pacientes con neumonía en China, 2019. N Engl J Med. [Revista en internet] 2020 [Citado, 4 Junio 2020];382: 727. 
16. Equipo de investigación de COVID-19. Características clínicas y virológicas de los primeros 12 pacientes con enfermedad por coronavirus 2019 (COVID-19) en los Estados Unidos. Nat Med. [Revista en internet] 2020 [Citado, 16 Mayo 2020].

17. $\mathrm{Hu} \mathrm{Z}$, Song $\mathrm{C}, \mathrm{Xu} \mathrm{C}$, et al. Características clínicas de 24 infecciones asintomáticas con COVID-19 examinadas entre contactos cercanos en Nanjing, China. Sci China Life Sci. [Revista en internet] 2020 [Citado, 13 Mayo 2020];63: 706.

18. Wang D, Hu B, Hu C, y col. Características clínicas de 138 pacientes hospitalizados con neumonía infectada con coronavirus novela 2019 en Wuhan, China. JAMA. [Revista en internet] 2020 [Citado, 5 Junio 2020].

19. Guan WJ, Ni ZY, Hu Y, et al. Características clínicas de la enfermedad por coronavirus 2019 en China. N Engl J Med. [Revista en internet] 2020 [Citado, 16 Junio 2020];382: 1708.

20. Chan JF, Yuan S, Kok KH, et al. Un grupo familiar de neumonía asociado con el nuevo coronavirus de 2019 que indica la transmisión de persona a persona: un estudio de un grupo familiar. Lancet. [Revista en internet] 2020 [Citado, 8 Junio 2020];395: 514.

21. Lauer SA, Grantz KH, Bi Q, et al. El período de incubación de la enfermedad por coronavirus 2019 (COVID-19) a partir de casos confirmados notificados públicamente: estimación y aplicación. Ann Intern Med. [Revista en internet] 2020 [Citado, 16 Junio 2020].

22. Huang C, Wang Y, Li X y col. Características clínicas de pacientes infectados con el nuevo coronavirus 2019 en Wuhan, China. Lancet. [Revista en internet] 2020 [Citado, 17 Junio 2020];395: 497.

23. Chen N, Zhou M, Dong X y col. Características epidemiológicas y clínicas de 99 casos de neumonía por coronavirus novedosa de 2019 en Wuhan, China: un estudio descriptivo. Lancet. [Revista en internet] 2020 [Citado, 15 Junio 2020];395:507.

24. Liu K, Fang YY, Deng Y, y col. Características clínicas de nuevos casos de coronavirus en hospitales terciarios de la provincia de Hubei. Chin Med J. [Revista en internet] 2020 [Citado, 4 Junio 2020];133:1025.

25. Yang X, Yu Y, Xu J, y cols. Curso clínico y resultados de pacientes críticamente enfermos con neumonía por SARS-CoV-2 en Wuhan, China: un estudio observacional, retrospectivo y de un solo centro. Lancet Respir Med. [Revista en internet] 2020 [Citado, 10 Junio 2020];8:475.

26. Wu Z, McGoogan JM. Características y lecciones importantes del brote de la enfermedad por coronavirus 2019 (COVID-19) en China: resumen de un informe de 72314 casos del Centro chino para el control y la prevención de enfermedades. JAMA. [Revista en internet] 2020 [Citado, 20 Mayo 2020].

27. Richardson S, Hirsch JS, Narasimhan M, y col. Presentando características, comorbilidades y resultados entre 5700 pacientes hospitalizados con COVID-19 en el área de la ciudad de Nueva York. JAMA. [Revista en internet] 2020 [Citado, 15 Junio 2020]

28. Chen T, Wu D, Chen H y col. Características clínicas de 113 pacientes fallecidos con enfermedad por coronavirus 2019: estudio retrospectivo. BMJ. [Revista en internet] 2020 [Citado, 6 Junio 2020];368:(09).

29. Cao Y, Li L, Feng Z, et al. Comparative genetic analysis of the novel coronavirus (2019$\mathrm{nCoV} / \mathrm{SARS}-\mathrm{CoV}-2)$ receptor ACE2 in different populations. Cell Discov. [Revista en internet] 2020 [Citado, 22 Mayo 2020];6:11. Disponible en: https://doi.org/10.1038/s4142 1-020-0147-1

30. Chen N, Zhou M, Dong X, et al. Epidemiological and clinical characteristics of 99 cases 
of 2019 novel coronavirus pneumonia in $\mathrm{Wu}-$ han, China: a descriptive study. Lancet. [Revista en internet] 2020 [Citado,11 Junio 2020]. Disponible en: https ://doi.org/10.1016/S0140 $-6736(20) 30211-7$.

31. Chen Z., Fu J., Shu Q., et al. Diagnosis and treatment recomendations for pediatric respiratory infection caused by the 2019 novel coronavirus. World J Pediatr. [Revista en internet] 2020 [Citado, 13 Junio 2020]. Disponible en: https:/ doi.org/10.1007/s12519-020-00345-5.

32. Lifen Y, Zhenyuan D, Mengqi D, et al. Sugerencias para el personal médico del de pediatría durante el tratamiento de 2019 - Infección por nCoV / neumonía. JN Med. [Revista en internet] 2020 [Citado, 26 Mayo 2020]. Disponible en: https://doi.org/10. 3969 / j.issn.0253 9802.2020.02.001.

33. Hu T, Fang L, Junling W, et al. Características clínicas del nuevo coronavirus 2019 (2019 $\mathrm{nCoV}$ ) infección en niños y prevención y control familiar. Med J Wuhan Univ. [Revista en internet] 2020 [Citado, 9 Junio 2020]. Disponible en: https://doi.org/10.14188 / j.1671 - 8852.2020.6020.

34. Feng K, Yun YX, Wang XF, y col. Primer caso de neumonía grave por coronavirus infantil en la infancia. Chin J Pediatr. [Revista en internet] 2020 [Citado, 16 Junio 2020];58(3):179183. Disponible en: https://doi.org/10.3760/ cma.j.issn.0578 - 1310.2020.03.003

35. Huang C, Wang Y, Li X, et al. Clinical features of patients infected with 2019 novel coronavirus in Wuhan, China. Lancet. [Revista en internet] 2020 [Citado, 13 Junio 2020]. Disponible en: https://doi.org/10.1016/S0140 -6736(20)30183-5.

36. Pathak, Elizabeth Barnett PhD, MSPH; Salemi, Jason L. PhD, MPH; Sobers, Natasha PhD, MBBS, MPH, FRSPH; Menard, Janelle PhD, $\mathrm{MPH}$; Hambleton, Ian R. PhD COVID-19 in Children in the United States, Journal of Public Health Management and Practice. [Revista en in- ternet] 2020 [Citado, 10 Junio 2020]. Disponible en: doi: 10.1097/PHH.0000000000001190

37. Coronavirus Disease 2019 in Children - United States, February 12-April 2, 2020. MMWR Morb Mortal Wkly Rep. ePub. [Revista en internet] 2020 [Citado, 4 Junio 2020]. Disponible en: https://www.cdc.gov/mmwr/volumes/69/wr/ mm6914e4.htm.

38. OMS National Health Commission of the People's Republic of China. China-World Health Organization joint study report on coronavirus disease 2019 (COVID-19). [Revista en internet] 2020 [Citado, 15 Junio 2020]. Disponible en: https://www.nhc.gov.cn/jkj/s3578/20200 2/87fd92510d094e4b9bad597608f5cc2c.shtml.

39. Bi Q, Wu Y, Mei S, et al. Epidemiología y transmisión de COVID-19 en Shenzhen, China: análisis de 391 casos y 1,286 de sus contactos cercanos. medRxiv. [Revista en internet] 2020 [Citado, 5 Junio 2020] Disponible en: 10.1101 / 2020.03.03.20028423.

40. Wu Z, McGoogan JM. Characteristics of and Important Lessons From the Coronavirus Disease 2019 (COVID-19) Outbreak in China: Summary of a Report of 72314 Cases From the Chinese Center for Disease Control and Prevention. JAMA. [Revista en internet] 2020 [Citado, 13 Junio 2020]

41. Livingston E, Bucher K. Coronavirus Disease 2019 (COVID-19) in Italy. JAMA. [Revista en internet] 2020 [Citado, 27 Mayo 2020]

42. DC COVID-19 Response Team. Coronavirus Disease 2019 in Children - United States, February 12-April 2, 2020. MMWR Morbidity and Mortality Weekly Report. ePub. [Revista en internet] 2020 [Citado, 5 Junio 2020]. Disponible en: http://dx.doi.org/10.15585/mmwr. mm6914e4external icon.

43. Tagarro A, Epalza C, Santos M, et al. Screening and Severity of Coronavirus Disease 2019 (COVID-19) in Children in Madrid, Spain. 
JAMA Pediatr. [Revista en internet] 2020 [Citado, 16 Junio 2020]

44. Qiu H, Wu J, Hong L, Luo Y, Song Q, Chen D. Clinical and epidemiological features of 36 children with coronavirus disease 2019 (COVID-19) in Zhejiang, China: an observational cohort study. Lancet Infect Dis. [Revista en internet] 2020 [Citado, 6 Junio 2020]

45. El Diario de Enfermedades Infecciosas Pediátricas. Infecciones por coronavirus en niños, incluido COVID-19 Una descripción general de la epidemiología, características clínicas, diagnóstico, tratamiento y opciones de prevención en niños. [Revista en internet] 2020 [Citado, 13 Junio 2020] (39);(5).

46. Kun Ling Shen, Yong Hong Yang, Rong Meng Jiang, Tian You Wang, · Dong Chi Zhao, Yi Jiang. Updated diagnosis, treatment and prevention of COVID 19 in children: experts' consensus statement (condensed version of the second edition). [Revista en internet] 2020 [Citado, 15 Mayo 2020]

47. Heng F, Liao C, Fan QH, et al. Clinical Characteristics of Children with Coronavirus Disease 2019 in Hubei, China. Curr Med Sci. [Revista en internet] 2020 [Citado, 11 Mayo 2020].

48. Xiaoxia Lu, M.D. Liqiong Zhang, M.D. Hui Du, M.D. Wuhan Children's Hospital. n engl j med. [Revista en internet] 2020 [Citado, 15 Junio 2020]382;(17). DOI: 10.1056/NEJMc2005073

49. Zhang H, Kang Z, Gong H. y col. El sistema digestivo es una ruta potencial para la infección 2019-nCov: un análisis bioinformático basado en transcriptomes unicelulares . BioRxiv 927806. [Revista en internet] 2020 [Citado, 10 Junio 2020] DOI: 10.1101 / 2020.01.30.927806

50. Tian, Yuan y col. "Artículo de revisión: características gastrointestinales en COVID-19 y la posibilidad de transmisión fecal". Farmacología alimentaria y terapéutica. [Revista en internet]
2020 [Citado, 13 Junio 2020];51:(9):843-851. DOI:10.1111/apt.15731

51. Hu T, Fang L, Junling W, et al. Características clínicas del nuevo coronavirus 2019 (2019 $\mathrm{nCoV}$ ) infección en niños y prevención y control familiar. Med J Wuhan Univ. [Revista en internet] 2020 [Citado, 12 Junio 2020]. Disponible en: https://doi.org/10. 14188 / j.1671 - 8852.2020.6020

52. Bi Q, Wu Y, Mei S, et al. Epidemiología y transmisión de COVID-19 en Shenzhen, China: análisis de 391 casos y 1,286 de sus contactos cercanos. medRxiv. [Revista en internet] 2020 [Citado, 15 Junio 2020]. Disponible en: 10.1101 / 2020.03.03.20028423. Consultado el 4 de marzo de 2020.

53. Chen H, Guo J, Wang C, et al. Características clínicas y potencial de transmisión vertical intrauterina de la infección por COVID-19 en nueve mujeres embarazadas: una revisión retrospectiva de los registros médicos. The Lancet . [Revista en internet] 2020 [Citado, 30 Mayo 2020]:1-7

54. Wang XF, Yuan J, Zheng YJ, et al. [Características clínicas y epidemiológicas de 34 niños con nueva infección por coronavirus 2019 en Shenzhen]. Zhonghua Er Ke Za Zhi . [Revista en internet] 2020 [Citado, 27 Mayo 2020]; 58 : E008.

55. Tiago H. de Souzaa, José A. Nadala, Roberto J. N. Nogueiraa, Ricardo M. Pereiraa, et al. Clinical Manifestations of Children with COVID-19: a Systematic Review. [Revista en internet] 2020 [Citado, 10 Junio 2020]

56. Toscano G, Palmerini F, Ravaglia $S$ et al (2000) Síndrome de Guillain-Barré asociado con SARS-CoV-2 N. Engl J. [Revista en internet] 2020 [Citado, 28 Mayo 2020]

57. Lechien JR, Chiesa-Estomba CM, De Siati DR, Horoi M, Le Bon SD, Rodriguez A. Disfunciones olfativas y gustativas como una presentación clínica de formas leves a moderadas de 
la enfermedad por coronavirus (COVID-19): a Estudio europeo multicéntrico. Eur Arch Otorhinolaryngol. [Revista en internet] 2020 [Citado, 22 Mayo 2020]. doi: 10.1007 / s00405-02005965-1.

58. Senanayake P, Drazba J, Shadrach K, Milsted A, Rungger-Brandle E, Nishiyama K. Angiotensin II y sus subtipos de receptores en la retina humana. Invest Ophthalmol Vis Sci. [Revista en internet] 2020 [Citado, 11 Junio 2020];48:33013311. doi: 10.1167 / iovs.06-1024

59. Wagner J, Jan Danser AH, Derkx FH, de Jong TV, Paul M, Mullins JJ. Demostración de ARNm de renina, ARNm de angiotensinógeno y expresión de ARNm de enzima convertidora de angiotensina en el ojo humano: evidencia de un sistema intraocular de renina-angiotensina. Fr. J. Ophthalmol. [Revista en internet] 2020 [Citado, 14 Junio 2020];80:159-163. doi: 10.1136 / bjo.80.2.159.

60. Wu P, Duan F, Luo C, Liu Q, Qu X, Liang $\mathrm{L}$, et al. Características de los hallazgos oculares de pacientes con enfermedad por coronavirus 2019 (COVID-19) en la provincia de Hubei, China. JAMA Ophthalmol. [Revista en internet] 2020 [Citado, 2 Junio 2020]

61. Riphagen S, Gomez X, Gonzalez-Martinez C, Wilkinson N, Theocharis P. Hyperinflammatory shock in children during COVID-19 pandemic. Lancet. [Revista en internet] 2020 [Citado, 3 Junio 2020]

62. Yun SH, Yang NR, Park SA. Associated symptoms of kawasaki disease. Korean Circ J 2011;41(7):394-398. 8. Baker AL, Lu M, Minich LL, et al. Associated symptoms in the ten days before diagnosis of Kawasaki disease. J Pediatr. [Revista en internet] 2020 [Citado, 6 Junio 2020];154(4):592-595.

63. Baker AL, Lu M, Minich LL, et al. Associated symptoms in the ten days before diagnosis of Kawasaki disease. J Pediatr. [Revista en internet] 2020 [Citado, 12 Mayo 2020];154(4):592595.

64. Mao L, Jin H, Wang M, et al. Manifesta- ciones neurológicas de pacientes hospitalizados con enfermedad por coronavirus 2019 en Wuhan, China . JAMA Neurol. [Revista en internet] 2020 [Citado, 15 Junio 2020].

65. Whittaker A, Anson M, Harky A. Manifestaciones neurológicas de COVID-19: una revisión sistemática y actualización actual. Acta Neurol Scand. [Revista en internet] 2020 [Citado, $17 \mathrm{Ju}$ nio 2020]: DOI:10.1111 / ane.13266. doi: 10.1111 / ane.13266. PMC7273036.

66. Cui J, Li F, Shi ZL. Origen y evolución de los coronavirus patógenos. Nat Rev Microbiol. UptoDate. [Revista en internet] 2020 [Citado, 3 Junio 2020];17:181 - 92). Disponible en: https:// doi.org/10.1038/s41579-018-0118-9 .

67. Wang XF, Yuan J, Zheng YJ, et al. [Características clínicas y epidemiológicas de 34 niños con nueva infección por coronavirus 2019 en Shenzhen]. Zhonghua Er Ke Za Zhi. UptoDate. [Revista en internet] 2020 [Citado, 23 Mayo 2020];58:E008.

68. Chen ZM, Fu JF, Shu Q, et al. Diagnóstico $\mathrm{y}$ recomendaciones de tratamiento para la infección respiratoria pediátrica causada por el nuevo coronavirus 2019. World J Pediatr. UptoDate. [Revista en internet] 2020 [Citado, 4 Junio 2020].

69. Wei M, Yuan J, Liu Y, et al. Nueva infección por coronavirus en bebés hospitalizados menores de 1 año en China. JAMA. [Revista en internet] 2020 [Citado, 16 Junio 2020]

70. Chan JF, Yuan S, Kok KH, et al. Un grupo familiar de neumonía asociado con el nuevo coronavirus de 2019 que indica la transmisión de persona a persona: un estudio de un grupo familiar. Lancet. [Revista en internet] 2020 [Citado, 26 Mayo 2020];395:514-523.

71. Zhu N, Zhang D, Wang W, Li X, Yang B, Song J, et al. Un nuevo coronavirus de pacientes con neumonía en China, 2019. N Engl J Med. [Revis- 
ta en internet] 2020 [Citado, 13 Junio 2020]:17. Disponible en: https://doi.org/10.1056/NEJMoa2001017.

72. Chen ZM, Fu JF, Shu Q, Chen YH, Hua CZ, Li FB, et al. Diagnóstico y recomendaciones de tratamiento para la infección respiratoria pediátrica causada por el nuevo coronavirus 2019. World J Pediatr. UptoDate. [Revista en internet] 2020 [Citado, 12 Junio 2020]. Disponible en: https://doi.org/10.1007/ s12519-020-00345-5.

73. Dong $\mathrm{Y}, \mathrm{Mo} \mathrm{X}, \mathrm{Hu} \mathrm{Y}$, Qi X, Jiang F, Jiang Z, et al. Características epidemiológicas de 2143 pacientes pediátricos con enfermedad por coronavirus 2019 en China epidemiología de COVID-19 entre niños en China. Pediatría. UptoDate. [Revista en internet] 2020 [Citado, 6 Junio 2020]. Disponible en: https://doi.org/10.1542/ peds.2020-0702.

74. Huang C, Wang Y, Li X y col. Características clínicas de pacientes infectados con el nuevo coronavirus 2019 en Wuhan, China. The lancet. [Revista en internet] 2020 [Citado, 15 Junio 2020]; 395(10223):497- 506.

75. Yang Y LQ, Liu M, y col. Características epidemiológicas y clínicas del nuevo brote de coronavirus de 2019 en China. MexRxiv. UptoDate. [Revista en internet] 2020 [Citado, 14 Junio 2020]

76. Wei M, Yuan J, Liu Y, Fu T, Yu X, Zhang Z. Nueva infección por coronavirus en niños hospitalizados menores de 1 año en China. JAMA [Revista en internet] 2020 [Citado, 5 Junio 2020];323 (13): 1313 - 1314.

77. Chen N, Zhou M, Dong X, et al. Características epidemiológicas y clínicas de 99 casos de neumonía por coronavirus novedosa en 2019 en Wuhan, China: un estudio descriptivo. The lancet. [Revista en internet] 2020 [Citado, 9 Junio 2020];395(10223): 507 - 513. 